\title{
On the lower bound of Spearman's footrule
}

https://doi.org/10.1515/demo-2019-0005

Received November 20, 2018; accepted April 7, 2019

Abstract: Úbeda-Flores showed that the range of multivariate Spearman's footrule for copulas of dimension $d \geq 2$ is contained in the interval $[-1 / d, 1]$, that the upper bound is attained exclusively by the upper FréchetHoeffding bound, and that the lower bound is sharp in the case where $d=2$. The present paper provides characterizations of the copulas attaining the lower bound of multivariate Spearman's footrule in terms of the copula measure but also via the copula's diagonal section.

Keywords: copulas, measures of dependence, sparse copulas, Spearman's footrule

MSC: $62 \mathrm{H} 05,62 \mathrm{H} 2 \mathrm{O}$

\section{Introduction}

Spearman's footrule is a "measure of disarray" (Diaconis and Graham [1]) which assigns to every copula $C$ : $[0,1]^{d} \rightarrow[0,1]$ a value $\phi(C)$ in the real numbers. Due to its "simplicity, robustness and natural interpretation" (Genest et al. [11]), it has already been used in several and quite different areas of research: This includes, for instance, genomics [12], information science [13] and economics [15]; we also refer to the impressive number of applications listed in [11] and [16].

Úbeda-Flores [18] showed that the range of Spearman's footrule for copulas of dimension $d \geq 2$ is contained in the interval $[-1 / d, 1]$, that the upper bound is attained exclusively by the upper Fréchet-Hoeffding bound, and that the lower bound is sharp in the case where $d=2$.

In the present paper, we show that, for every dimension $d \geq 2$, the lower bound of Spearman's footrule is sharp and attained by uncountably many copulas. To this end, we first provide characterizations of the copulas attaining the lower bound of Spearman's footrule. It turns out that a copula minimizes Spearman's footrule if and only if the support of the corresponding copula measure is contained in the complement of the set $\left(0, \frac{d-1}{d}\right)^{d} \cup\left(\frac{1}{d}, 1\right)^{d}$. We then construct a sparse structure and show that every sparse copula having this sparse structure minimizes Spearman's footrule. This then leads to uncountably many copulas attaining the lower bound.

The paper is organized as follows: Section 2 collects some definitions and results on copulas and related topics which will be needed in this paper. Section 3 gives a brief discussion of Spearman's footrule and provides several characterizations of the copulas attaining the lower bound; it turns out that it is important where the mass of the copula is distributed, not how. In Section 4 we then outline the concept of sparse copulas and provide a class of sparse copulas minimizing Spearman's footrule in any dimension.

^Corresponding Author: Sebastian Fuchs: Technische Universität Dortmund, E-mail: sfuchs@statistik.tu-dortmund.de Yann McCord: Technische Universität Dresden, E-mail: yannmccord@gmail.com 


\section{Preliminaries}

Let $\mathbb{I}:=[0,1]$ and let $d \geq 2$ be an integer which will be kept fix throughout this paper. We denote by $\mathbf{0}$ the vector in $\mathbb{R}^{d}$ with all coordinates being equal to 0 and by $\mathbf{1}$ the vector in $\mathbb{R}^{d}$ with all coordinates being equal to 1. In the sequel, we denote by $\mathcal{C}$ the collection of all $d$-dimensional copulas; for details on copulas we refer to $[4,14]$. Since every copula $C \in \mathcal{C}$ is continuous and has a unique extension to a distribution function $\mathbb{R}^{d} \rightarrow \mathbb{I}$, there exists a unique probability measure $Q^{C}: \mathcal{B}\left(\mathbb{I}^{d}\right) \rightarrow \mathbb{I}$ satisfying $Q^{C}[[\mathbf{0}, \mathbf{u}]]=Q^{C}[(\mathbf{0}, \mathbf{u})]=C(\mathbf{u})$ for every $\mathbf{u} \in \mathbb{I}^{d}$. The probability measure $Q^{C}$ is said to be the copula measure corresponding to $C$.

For $i, j \in\{1, \ldots, d\}$ with $i<j$, we define the maps $\pi_{i, j}: \mathcal{C} \rightarrow \mathcal{C}$ by letting

$$
\left(\pi_{i, j}(C)\right)(\mathbf{u}):=C\left(u_{1}, \ldots, u_{i-1}, u_{j}, u_{i+1}, \ldots, u_{j-1}, u_{i}, u_{j+1}, \ldots, u_{d}\right)
$$

Each of these permutations is an involution. For the sake of simplicity, we further define the map $\tilde{\tau}: \mathbb{I}^{d} \rightarrow \mathbb{I}^{d}$ and the map $\tau: \mathcal{C} \rightarrow \mathcal{C}$ by letting

$$
\tilde{\tau}(\mathbf{u}):=\mathbf{1}-\mathbf{u} \quad \text { and } \quad(\tau(C))(\mathbf{u}):=\left(Q^{C}\right)_{\tilde{\tau}}[[\mathbf{0}, \mathbf{u}]]
$$

We note that the total reflection $\tau$ transforms every copula into its survival copula. We refer to $[6,10]$ for further details on permutations and the total reflection.

The total reflection also provides an order relation $\preceq$ on $\mathcal{C}$ which is called the concordance order on $\mathcal{C}$ and is defined by setting $C \preceq D$ if and only if $C(\mathbf{u}) \leq D(\mathbf{u})$ and $(\tau(C))(\mathbf{u}) \leq(\tau(D))(\mathbf{u})$ for every $\mathbf{u} \in \mathbb{I}^{d}$. Since $\tau(M)=M$, the upper Fréchet-Hoeffding bound $M$ is the greatest element in (e, $\preceq)$; similarly, in the case $d=2$, the lower Fréchet-Hoeffding bound $W$ is the least element in $(\mathcal{C}, \preceq)$.

\section{Spearman's Footrule}

In this section we provide characterizations of the copulas attaining the lower bound of multivariates Spearman's footrule. The key for dealing with Spearman's footrule of a copula turns out to be the copula's diagonal section.

For a map $C: \mathbb{I}^{d} \rightarrow \mathbb{I}$, the map $\delta_{C}: \mathbb{I} \rightarrow \mathbb{I}$ given by

$$
\delta_{C}(u):=C(u \mathbf{1})
$$

is called the diagonal section of $C$. The following result may be found in [4, Theorem 2.6.1]:

3.1 Proposition. A function $\delta: \mathbb{I} \rightarrow \mathbb{I}$ is the diagonal section of a copula $C \in \mathcal{C}$ if and only if it satisfies the following properties

(i) $\delta(0)=0$ and $\delta(1)=1$.

(ii) $\delta$ is increasing.

(iii) The inequality $\delta(t) \leq t$ holds for all $t \in \mathbb{I}$.

(iv) The inequality $|\delta(t)-\delta(s)| \leq d|t-s|$ holds for all $s, t \in \mathbb{I}$.

The diagonal section $\delta_{W}$ of the $d$-dimensional lower Fréchet-Hoeffding bound $W$ satisfies

$$
\delta_{W}(u)=\max \{d u-(d-1), 0\}
$$

and $\delta_{W} \leq \delta_{C}$ for every $d$-dimensional copula $C$. Although $W$ fails to be a copula whenever $d \geq 3$, due to Proposition 3.1, for every dimension $d \geq 2$ there exists some copula $C$ satisfying $\delta_{C}=\delta_{W}$. 
The map $\phi: \mathcal{C} \rightarrow \mathbb{R}$ given by

$$
\begin{aligned}
\phi(C) & :=\frac{d+1}{d-1} \int_{\mathbb{I}} \delta_{C}(u)+\delta_{\tau(C)}(u) \mathrm{d} \lambda(u)-\frac{2}{d-1} \\
& =\frac{2(d+1)}{d-1} \int_{\mathbb{I}^{d}} \frac{C(\mathbf{u})+(\tau(C))(\mathbf{u})}{2} \mathrm{~d} Q^{M}(\mathbf{u})-\frac{2}{d-1}
\end{aligned}
$$

is called Spearman's footrule; here, $\lambda$ denotes the restriction of the Lebesgue measure to the Borel $\sigma$-field $\mathcal{B}(\mathbb{I})$ on $\mathbb{I}$. This definition of Spearman's footrule is in accordance with the definitions used in [11, 18]. It is immediate from [7] and [18] that $\phi$ is

- $\quad$ symmetric, i.e. the identity $\phi\left(\pi_{i, j}(C)\right)=\phi(C)$ holds for every $C \in \mathcal{C}$ and all $i, j \in\{1, \ldots, d\}$ with $i<j$;

- $\quad$ radially symmetric, i.e. the identity $\phi(\tau(C))=\phi(C)$ holds for every $C \in \mathcal{C}$;

- $\quad$ concordance order preserving, i.e. the inequality $\kappa(C) \leq \kappa(D)$ holds for all $C, D \in \mathcal{C}$ satisfying $C \preceq D$;

- continuous, i.e. for any sequence $\left\{C_{n}\right\}_{n \in \mathbb{N}} \subseteq \mathcal{C}$ and any $C \in \mathcal{C}$, uniform convergence $\lim _{n \rightarrow \infty} C_{n}=C$ implies $\lim _{n \rightarrow \infty} \kappa\left(C_{n}\right)=\kappa(C)$; and satisfies

- $\phi(\Pi)=0$, where $\Pi$ denotes the product copula.

Note that $\phi$ is not a measure of concordance in the sense of $[2,8,17]$. For more details on Spearman's footrule we refer to $[11,18]$ and the references there.

Úbeda-Flores [18] showed that the inequalities

$$
-\frac{1}{d} \leq \phi(C) \leq 1
$$

hold for every copula $C$ of dimension $d \geq 2$, that the upper bound is attained exclusively by the upper FréchetHoeffding bound $M$, and that the lower bound is sharp in the case where $d=2$ and is attained by the lower Fréchet-Hoeffding bound $W$; see [18, Theorem 4.2].

In the sequel, we first characterize the class of all those copulas satisfying $\phi(C)=-\frac{1}{d}$ in terms of their copula measure but also via the copula's diagonal section. In Section 4, we then show that this class is non-empty and contains uncountably many copulas. This implies that the above lower bound of Spearman's footrule is sharp and is attained by uncountably many copulas.

3.2 Theorem. For a copula $C \in \mathcal{C}$ the following statements are equivalent:

(a) $\phi(C)=-\frac{1}{d}$.

(b) $\delta_{C}=\delta_{\tau(C)}=\delta_{W}$.

(c) $C$ satisfies $Q^{C}\left[\left[0, \frac{d-1}{d}\right]^{d}\right]=0$ and $Q^{C}\left[\left[\frac{1}{d}, 1\right]^{d}\right]=0$.

Proof. The equivalence of (a) and (b) is straightforward. Note that $\delta_{C}\left(\frac{d-1}{d}\right)=Q^{C}\left[\left[0, \frac{d-1}{d}\right]^{d}\right]$ and

$$
\delta_{\tau(C)}\left(\frac{d-1}{d}\right)=\left(Q^{C}\right)_{\tilde{\tau}}\left[\left[0, \frac{d-1}{d}\right]^{d}\right]=Q^{C}\left[\left[\frac{1}{d}, 1\right]^{d}\right]
$$

Thus, $\delta_{C}(u)=0=\delta_{\tau(C)}(u)$ holds for all $u \in\left[0, \frac{d-1}{d}\right]$ if and only if $Q^{C}\left[\left[0, \frac{d-1}{d}\right]^{d}\right]=0=Q^{C}\left[\left[\frac{1}{d}, 1\right]^{d}\right]$; in this case, Lipschitz continuity of a copula's diagonal section (Proposition 3.1 (iv)) yields, for every $u \in\left(\frac{d-1}{d}, 1\right]$,

$$
\delta_{C}(u)=\delta_{C}(u)-\delta_{C}\left(\frac{d-1}{d}\right) \leq d\left(u-\frac{d-1}{d}\right)=d u-(d-1)
$$

and

$$
1-\delta_{C}(u)=\delta_{C}(1)-\delta_{C}(u) \leq d(1-u)
$$

and hence $\delta_{C}(u)=d u-(d-1)=\delta_{W}(u)$ for every $u \in\left(\frac{d-1}{d}, 1\right]$, and the same argument applies to $\delta_{\tau(C)}$ in the place of $\delta_{C}$. This proves the equivalence of (b) and (c). 
3.3 Remark. Condition (c) in Theorem 3.2 implies that a copula $C$ minimizes Spearman's footrule if and only if $C\left(\frac{d-1}{d} \mathbf{1}\right)=0$ and $(\tau(C))\left(\frac{d-1}{d} \mathbf{1}\right)=0$, i.e. minimality of Spearman's footrule is a property of the copula's and its survival copula's values at certain points in the unit hypercube. At this point, it is worth to mention that a qualitatively similar property characterizes minimality of multivariate Kendall's tau; see [9, Theorem 3.4].

Note, that condition (c) in Theorem 3.2 solely depends on where the mass of the copula is distributed, not how. We illustrate this result for the cases $d \in\{2,3\}$ :

\subsection{Examples.}

(1) In the bivariate case, the mass of a copula $C$ minimizing Spearman's footrule needs to lie in the upper left and lower right rectangles below (grey area)

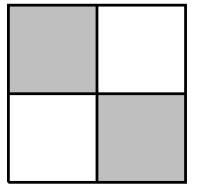

(2) In the trivariate case, the support of the corresponding copula measure $Q^{C}$ needs to be a subset of the below 12 grey subcubes

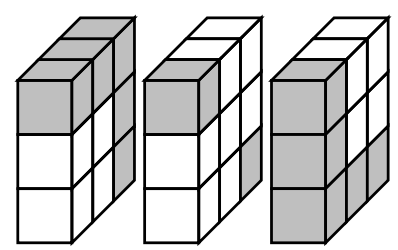

In the next section we discuss a copula construction principle that fits quite well with condition (c) in Theorem 3.2. In passing, we prove the existence of a copula $C$ minimizing Spearman's footrule and show that Spearman's footrule is minimized by uncountably many copulas.

\section{Sparse Copulas minimizing Spearman's Footrule}

In this section we outline the concept of sparse copulas. In particular, we construct a certain sparse structure and show that every sparse copula having this sparse structure minimizes Spearman's footrule.

Consider an index set $J \subseteq \mathbb{N}$ such that $|J| \geq 2$. A family $\left\{\left[\mathbf{a}_{n}, \mathbf{b}_{n}\right]\right\}_{n \in J}$ of non-empty closed intervals in $\mathbb{I}^{d}$ is called a sparse structure, if it satisfies the following conditions:

(i) $b_{n, i}-a_{n, i}=b_{n, j}-a_{n, j}$ holds for every $n \in J$ and all $i, j \in\{1, \ldots, d\}$.

(ii) $\left(a_{m, i}, b_{m, i}\right) \cap\left(a_{n, i}, b_{n, i}\right)=\emptyset$ holds for all $m, n \in J$ such that $m \neq n$ and for every $i \in\{1, \ldots, d\}$.

(iii) $\sum_{n \in J}\left(b_{n, i}-a_{n, i}\right)=1$ holds for every $i \in\{1, \ldots, d\}$.

This definition is due to [3, Definition 2.1] and [4, Section 3.7].

For a sparse structure $\mathbf{S}=\left\{\left[\mathbf{a}_{n}, \mathbf{b}_{n}\right]\right\}_{n \in J}$ and every $n \in J$, we define the map $\psi_{n}^{\mathbf{S}}: \mathbb{I}^{d} \rightarrow\left[\mathbf{a}_{n}, \mathbf{b}_{n}\right]$ by letting

$$
\psi_{n}^{\mathbf{S}}(\mathbf{u}):=\left(a_{n, 1}+\left(b_{n, 1}-a_{n, 1}\right) u_{1}, \ldots, a_{n, d}+\left(b_{n, d}-a_{n, d}\right) u_{d}\right)
$$

and, for a family of copulas $\mathbf{D}=\left\{D_{n}\right\}_{n \in J}$, we define the map $C_{(\mathbf{S}, \mathbf{D})}: \mathbb{I}^{d} \rightarrow \mathbb{I}$ by letting

$$
C_{(\mathbf{S}, \mathbf{D})}(\mathbf{u}):=\sum_{n \in J}\left(b_{n, 1}-a_{n, 1}\right) D_{n}\left(\left(\frac{u_{1}-a_{n, 1}}{b_{n, 1}-a_{n, 1}} \wedge 1\right)^{+}, \ldots,\left(\frac{u_{d}-a_{n, d}}{b_{n, d}-a_{n, d}} \wedge 1\right)^{+}\right)
$$

where $\wedge$ denotes the minimum and $x^{+}$the positive part of the real number $x \in \mathbb{R}$. The map $C_{(\mathbf{S}, \mathbf{D})}$ is called the sparse copula of $\mathbf{D}$ with respect to $\mathbf{S}$. The following result is due to [3, Propositions 2.1 and 2.2]: 
4.1 Proposition. For any sparse structure $\mathbf{S}=\left\{\left[\mathbf{a}_{n}, \mathbf{b}_{n}\right]\right\}_{n \in J}$ and any family of copulas $\mathbf{D}=\left\{D_{n}\right\}_{n \in J}$, the sparse copula of $\mathbf{D}$ with respect to $\mathbf{S}$ is a copula and its copula measure satisfies

$$
Q^{C_{(\mathbf{s}, \mathbf{D})}}[B]=\sum_{n \in J}\left(b_{n, 1}-a_{n, 1}\right)\left(Q^{D_{n}}\right)_{\psi_{n}^{\mathbf{s}}}\left[B \cap\left[\mathbf{a}_{n}, \mathbf{b}_{n}\right]\right]
$$

It turns out that the total reflection of any sparse copula is again a sparse copula:

4.2 Theorem. For any sparse structure $\mathbf{S}=\left\{\left[\mathbf{a}_{n}, \mathbf{b}_{n}\right]\right\}_{n \in J}$ and any family of copulas $\mathbf{D}=\left\{D_{n}\right\}_{n \in J}$, the total reflection of $C_{(\mathbf{S}, \mathbf{D})}$ is again a sparse copula satisfying

$$
\tau\left(C_{(\mathbf{S}, \mathbf{D})}\right)=C_{(\tilde{\tau}(\mathbf{S}), \tau(\mathbf{D}))}
$$

where $\tilde{\tau}(\mathbf{S})=\left\{\left[\mathbf{1}-\mathbf{b}_{n}, \mathbf{1}-\mathbf{a}_{n}\right]\right\}_{n \in J}$ and $\tau(\mathbf{D})=\left\{\tau\left(D_{n}\right)\right\}_{n \in J}$.

Proof. For every $\mathbf{u} \in \mathbb{I}^{d}$ we first have

$$
\begin{aligned}
\left(\tilde{\tau} \circ \psi_{n}^{\mathbf{S}}\right)(\mathbf{u}) & =\mathbf{1}-\left(a_{n, 1}+\left(b_{n, 1}-a_{n, 1}\right) u_{1}, \ldots, a_{n, d}+\left(b_{n, d}-a_{n, d}\right) u_{d}\right) \\
& =\left(1-a_{n, 1}-\left(b_{n, 1}-a_{n, 1}\right) u_{1}, \ldots, 1-a_{n, d}-\left(b_{n, d}-a_{n, d}\right) u_{d}\right) \\
& =\left(\left(1-b_{n, 1}\right)+\left(\left(1-a_{n, 1}\right)-\left(1-b_{n, 1}\right)\right)\left(1-u_{1}\right), \ldots,\left(1-b_{n, d}\right)+\left(\left(1-a_{n, d}\right)-\left(1-b_{n, d}\right)\right)\left(1-u_{d}\right)\right) \\
& =\left(\psi_{n}^{\tilde{\tau}(\mathbf{S})} \circ \tilde{\tau}\right)(\mathbf{u})
\end{aligned}
$$

The previous identity together with Proposition 4.1 then yields

$$
\begin{aligned}
& \left(\tau\left(C_{(\mathbf{S}, \mathbf{D})}\right)\right)(\mathbf{u})=\left(Q^{C_{(\mathbf{s}, \mathbf{D})}}\right)_{\tilde{\tau}}[[\mathbf{0}, \mathbf{u}]] \\
& =Q^{C_{(\mathbf{s}, \mathbf{D})}}[[\mathbf{1}-\mathbf{u}, \mathbf{1}]] \\
& \left.=\sum_{n \in J}\left(b_{n, 1}-a_{n, 1}\right)\left(Q^{D_{n}}\right)_{\psi_{n}^{\mathrm{s}}}[\mathbf{1}-\mathbf{u}, \mathbf{1}] \cap\left[\mathbf{a}_{n}, \mathbf{b}_{n}\right]\right] \\
& =\sum_{n \in J}\left(b_{n, 1}-a_{n, 1}\right)\left(Q^{D_{n}}\right)_{\tilde{\tau} \circ \psi_{n}^{\mathbf{S}}}\left[[\mathbf{0}, \mathbf{u}] \cap\left[\mathbf{1}-\mathbf{b}_{n}, \mathbf{1}-\mathbf{a}_{n}\right]\right] \\
& =\sum_{n \in J}\left(b_{n, 1}-a_{n, 1}\right)\left(Q^{D_{n}}\right)_{\psi_{n}^{\tilde{\tau} \mathbf{( s )}} \circ \tilde{\tau}}\left[[\mathbf{0}, \mathbf{u}] \cap\left[\mathbf{1}-\mathbf{b}_{n}, \mathbf{1}-\mathbf{a}_{n}\right]\right] \\
& =\sum_{n \in J}\left(\left(1-a_{n, 1}\right)-\left(1-b_{n, 1}\right)\right)\left(Q^{\tau\left(D_{n}\right)}\right)_{\psi_{n}^{\tilde{\tau} \mathbf{s})}}\left[[\mathbf{0}, \mathbf{u}] \cap\left[\mathbf{1}-\mathbf{b}_{n}, \mathbf{1}-\mathbf{a}_{n}\right]\right] \\
& =\left(C_{(\tilde{\tau}(\mathbf{S}), \tau(\mathbf{D}))}\right)(\mathbf{u})
\end{aligned}
$$

for all $\mathbf{u} \in \mathbb{I}^{d}$. This proves the assertion.

Any sparse copula having the below sparse structure minimizes Spearman's footrule. Thus, again, for minimizing Spearman's footrule it is important where the mass of the copula is distributed, not how.

4.3 Theorem. Consider $J=\{1, \ldots, d\}$ and a sparse structure $\mathbf{S}=\left\{\left[\mathbf{a}_{n}, \mathbf{b}_{n}\right]\right\}_{n \in J}$ satisfying $b_{n, 1}-a_{n, 1}=1 / d$ and $\left\{a_{n, 1}, \ldots, a_{n, d}\right\}=\{0,1 / d, \ldots,(d-1) / d\}$ for all $n \in J$. Then the sparse copula of any $\mathbf{D}$ with respect to $\mathbf{S}$ minimizes Spearman's footrule.

Proof. Since $\left\{a_{n, 1}, \ldots, a_{n, d}\right\}=\{0,1 / d, \ldots,(d-1) / d\}$ for all $n \in J$, none of the $d$ intervals $\left[\mathbf{a}_{n}, \mathbf{b}_{n}\right]$ and none of the $d$ intervals $\left[\mathbf{1}-\mathbf{b}_{n}, \mathbf{1}-\mathbf{a}_{n}\right]$ is contained in $\left(0, \frac{d-1}{d}\right)^{d}$, i.e. $\left(0, \frac{d-1}{d}\right)^{d} \cap\left[\mathbf{a}_{n}, \mathbf{b}_{n}\right]=\emptyset$ and $\left(0, \frac{d-1}{d}\right)^{d} \cap[\mathbf{1}-$ $\left.\mathbf{b}_{n}, \mathbf{1}-\mathbf{a}_{n}\right]=\emptyset$. Applying Proposition 4.1 we hence obtain

$$
Q^{C_{(\mathbf{s}, \mathbf{D})}}\left[\left[0, \frac{d-1}{d}\right]^{d}\right]=Q^{C_{(\mathbf{s}, \mathbf{D})}}\left[\left(0, \frac{d-1}{d}\right)^{d}\right]=\sum_{n \in J}\left(b_{n, 1}-a_{n, 1}\right)\left(Q^{D_{n}}\right)_{\psi_{n}^{\mathbf{s}}}\left[\left(0, \frac{d-1}{d}\right)^{d} \cap\left[\mathbf{a}_{n}, \mathbf{b}_{n}\right]\right]=0
$$


and Theorem 4.2 together with Proposition 4.1 yields

$$
\begin{aligned}
Q^{C_{(\mathbf{S}, \mathbf{D})}\left[\left[\frac{1}{d}, 1\right]^{d}\right]} & =Q^{\tau\left(C_{(\mathbf{S}, \mathbf{D})}\right)}\left[\left[0, \frac{d-1}{d}\right]^{d}\right] \\
& =Q^{C_{(\bar{\tau}(\mathbf{S}), \tau(\mathbf{D}))}}\left[\left[0, \frac{d-1}{d}\right]^{d}\right] \\
& =Q^{C_{(\tilde{\tau}(\mathbf{S}) \tau(\mathbf{D}))}}\left[\left(0, \frac{d-1}{d}\right)^{d}\right] \\
& =\sum_{n \in J}\left(\left(1-a_{n, 1}\right)-\left(1-b_{n, 1}\right)\right)\left(Q^{\tau\left(D_{n}\right)}\right)_{\psi_{n}^{\tilde{\tau}(\mathbf{S})}}\left[\left(0, \frac{d-1}{d}\right)^{d} \cap\left[\mathbf{1}-\mathbf{b}_{n}, \mathbf{1}-\mathbf{a}_{n}\right]\right] \\
& =0
\end{aligned}
$$

Thus, by Theorem 3.2, $C_{(\mathbf{S}, \mathbf{D})}$ minimizes Spearman's footrule.

Finally, we apply Theorem 4.3 to the cases $d \in\{2,3,4\}$. In particular, we show that any sparse copula of dimension $d=2$ having the same sparse structure than the lower Fréchet-Hoeffding bound $W$ is a minimizer of Spearman's footrule:

\subsection{Examples.}

(1) In the case $d=2$, the only sparse structure satisfying the assumptions of Theorem 4.3 is the sparse structure $\mathbf{S}=\left\{\left[\mathbf{a}_{i}, \mathbf{b}_{i}\right]\right\}_{i \in\{1,2\}}$ with

$$
\begin{aligned}
& \mathbf{a}_{1}=(0,1 / 2) \quad \mathbf{b}_{1}=(1 / 2,1) \\
& \mathbf{a}_{2}=(1 / 2,0) \quad \mathbf{b}_{2}=(1,1 / 2)
\end{aligned}
$$

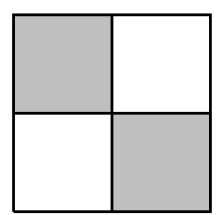

(2) In the trivariate case, the only two possible sparse structures satisfying the assumptions of Theorem 4.3 are the sparse structures $\mathbf{S}_{1}=\left\{\left[\mathbf{a}_{1, i}, \mathbf{b}_{1, i}\right]\right\}_{i \in\{1,2,3\}}$ with

$$
\begin{aligned}
& \mathbf{a}_{1,1}=(0,1 / 3,2 / 3) \quad \mathbf{b}_{1,1}=(1 / 3,2 / 3,1) \\
& \mathbf{a}_{1,2}=(1 / 3,2 / 3, \quad 0) \quad \mathbf{b}_{1,2}=(2 / 3,1,1 / 3) \\
& \mathbf{a}_{1,3}=(2 / 3, \quad 0,1 / 3) \quad \mathbf{b}_{1,3}=(1,1 / 3,2 / 3)
\end{aligned}
$$

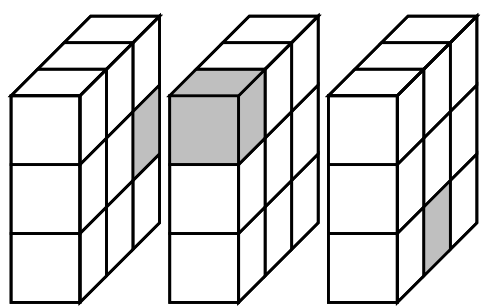

and $\mathbf{S}_{2}=\left\{\left[\mathbf{a}_{2, i}, \mathbf{b}_{2, i}\right]\right\}_{i \in\{1,2,3\}}$ with

$$
\begin{aligned}
& \mathbf{a}_{2,1}=(0,2 / 3,1 / 3) \quad \mathbf{b}_{2,1}=(1 / 3,1,2 / 3) \\
& \mathbf{a}_{2,2}=(1 / 3,0,2 / 3) \quad \mathbf{b}_{2,2}=(2 / 3,1 / 3,1) \\
& \mathbf{a}_{2,3}=(2 / 3,1 / 3, \quad 0) \quad \mathbf{b}_{2,3}=(1,2 / 3,1 / 3)
\end{aligned}
$$

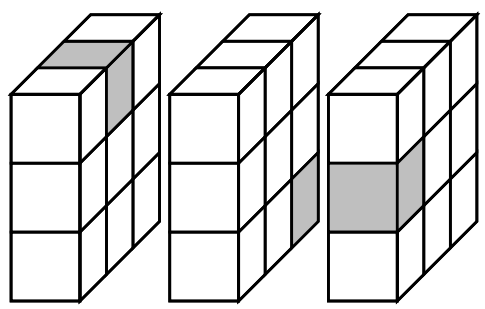


(3) In the case $d=4$, there exist exactly twelve possible sparse structures satisfying the assumptions of Theorem 4.3

The above example (1) ensures that even in the case $d=2$ there exist uncountably many copulas minimizing Spearman's footrule.

4.5 Remark. The sparse copulas provided in Theorem 4.3 all minimize Spearman's footrule. However, obviously, there also exist other copulas satisfying condition (c) in Theorem 3.2. Further minimizers of Spearman's footrule may be obtained applying sparse structures with a finer mesh $J$ satisfying $b_{n, 1}-a_{n, 1}=1 /|J|$, but also those copulas induced by certain generalized transformation matrices studied in [5, Section 5]. In contrast to sparse copulas, the latter construction principle allows distributing some mass to each single subcube in the complement of the set $\left[0, \frac{d-1}{d}\right]^{d} \cup\left[\frac{1}{d}, 1\right]^{d}$, however, the mass assigned to each of these subcubes has to be distributed in exactly the same way.

Acknowledgement: The authors are most grateful to the referees whose thoughtful comments led to a more comprehensive discussion of the subject.

\section{References}

[1] Diaconis, P. and R. L. Graham (1977). Spearman's footrule as a measure of disarray. J. R. Stat. Soc. Ser. B. Stat. Methodol. 39(2), 262-268.

[2] Dolati, A. and M. Úbeda-Flores (2006). On measures of multivariate concordance. J. Probab. Stat. Sci. 4(2), 147-163.

[3] Durante, F. and J. Fernández-Sánchez (2010). Multivariate shuffles and approximation of copulas. Statist. Probab. Lett. 80(22-23), 1827-1834.

[4] Durante, F. and C. Sempi (2016). Principles of Copula Theory. CRC Press, Boca Raton FL.

[5] Fernández-Sánchez, J. and W. Trutschnig (2015). Conditioning-based metrics on the space of multivariate copulas and their interrelation with uniform and levelwise convergence and iterated function systems. J. Theoret. Probab. 28(4), 1311-1336.

[6] Fuchs, S. (2014). Multivariate copulas: Transformations, symmetry, order and measures of concordance. Kybernetika 50(5), 725-743.

[7] Fuchs, S. (2016a). A biconvex form for copulas. Depend. Model. 4, 63-75.

[8] Fuchs, S. (2016b). Copula-induced measures of concordance. Depend. Model. 4, 205-214.

[9] Fuchs, S., Y. McCord, and K. D. Schmidt (2018). Characterizations of copulas attaining the bounds of multivariate Kendall's tau. J. Optim. Theory Appl. 178(2), 424-438.

[10] Fuchs, S. and K. D. Schmidt (2014). Bivariate copulas: Transformations, asymmetry and measures of concordance. Kybernetika 50(1), 109-125.

[11] Genest, C., J. Nešlehová, and N. Ben Ghorbal (2010). Spearman's footrule and Gini's gamma: a review with complements. J. Nonparametr. Stat. 22(8), 937-954.

[12] Kim, B. S., S. Y. Rha, G. B. Cho, and H. C. Chung (2004). Spearman's footrule as a measure of cDNA microarray reproducibility. Genomics 84(2), 441-448.

[13] Lee, P. H. and P. L. H. Yu (2010). Distance-based tree models for ranking data. Comput. Statist. Data Anal. 54(6), $1672-1682$.

[14] Nelsen, R. B. (2006). An Introduction to Copulas. Second edition. Springer, New York.

[15] Pérez, A. and M. Prieto-Alaiz (2016). Measuring the dependence among dimensions of welfare: A study based on Spearman's footrule and Gini's gamma. Internat. J. Uncertain. Fuzziness Knowledge-Based Systems 24(1), 87-105.

[16] Sen, P. K., I. A. Salama, and D. Quade (2011). Spearman's footrule: Asymptotics in applications. Chil. J. Stat. 2(1), 3-20.

[17] Taylor, M. D. (2016). Multivariate measures of concordance for copulas and their marginals. Depend. Model. 4, $224-236$.

[18] Úbeda-Flores, M. (2005). Multivariate versions of Blomqvist's beta and Spearman's footrule. Ann. Inst. Statist. Math. 57(4), 781-788. 